\title{
Fixed point results for weak contractions in partially ordered $b$-metric space
}

\author{
N. Seshagiri Rao ${ }^{1 *}$, K. Kalyani ${ }^{2}$ and K. Prasad ${ }^{3}$
}

\begin{abstract}
Objectives: We explore the existence of a fixed point as well as the uniqueness of a mapping in an ordered $b$-metric space using a generalized $(\breve{\psi}, \hat{\eta})$-weak contraction. In addition, some results are posed on a coincidence point and a coupled coincidence point of two mappings under the same contraction condition. These findings generalize and build on a few recent studies in the literature. At the end, we provided some examples to back up our findings.

Result: In partially ordered $b$-metric spaces, it is discussed how to obtain a fixed point and its uniqueness of a mapping, and also investigated the existence of a coincidence point and a coupled coincidence point for two mappings that satisfying generalized weak contraction conditions.

Keywords: $(\check{\psi}, \hat{\eta})$-weak contraction, Fixed point, Coincidence and coupled coincidence points, Ordered $b$-metric space
\end{abstract}

Mathematics Subject Classification: $54 \mathrm{H} 25,47 \mathrm{H} 10$

\section{Introduction}

In a wide range of pure and applied mathematics problems, fixed points of mappings that satisfy contractive conditions in extended metric spaces are extremely useful. First, Ran and Reuings [32] described the existence of fixed points in this direction for certain maps in ordered metric space and exhibited matrix linear equations applications. Following that, Nieto et al. [28, 29] expanded the result of [32] to nondecreasing mappings and used their findings to obtain differential equations solutions. Agarwal et al. [3] and O'Regan et al. [30] examined the influence of generalized contractions in ordered spaces at the same time. Bhaskar and Lakshmikantham [11] first developed coupled fixed point theory for some maps, then used the results to find a unique solution to periodic boundary value problems. Following that,

*Correspondence: seshu.namana@gmail.com

1 Department of Applied Mathematics, School of Applied Natural

Sciences, Adama Science and Technology University, Post Box No.1888,

Adama, Ethiopia

Full list of author information is available at the end of the article
Lakshmikantham and Ćirić [22], which were the extensions of [11] involving monotone property to a function in the space, pioneered the idea of coupled coincidence, common fixed point results. [19, 25, 34-37] provide additional information on coupled fixed point effects in various spaces under various contractive conditions.

A $b$-metric space is one of several generalizations of a standard metric space proposed by Bakhtin in his work [9], and widely used by Czerwik in his work [14, 15]. Following that, a lot of progress was made in acquiring the results of fixed points to single valued as well as multivalued operators in the space, as evidenced by $[1,2,4-8$, $10,13,16-18,20,21,23,24,26,27,31,38-41]$.

We demonstrate some fixed points results for mappings in ordered $b$-metric space that satisfy a generalized weak contraction in this paper. The results from $[10,11$, $19,22,33$ ] are expanded here as well as some examples noted to support the findings at the end of our work.

\section{Preliminaries}

The following definitions are subsequently used in our study.

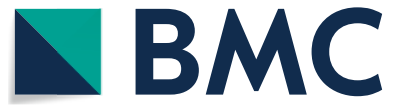

(c) The Author(s) 2021. This article is licensed under a Creative Commons Attribution 4.0 International License, which permits use, sharing, adaptation, distribution and reproduction in any medium or format, as long as you give appropriate credit to the original author(s) and the source, provide a link to the Creative Commons licence, and indicate if changes were made. The images or other third party material in this article are included in the article's Creative Commons licence, unless indicated otherwise in a credit line to the material. If material is not included in the article's Creative Commons licence and your intended use is not permitted by statutory regulation or exceeds the permitted use, you will need to obtain permission directly from the copyright holder. To view a copy of this licence, visit http://creativeco mmons.org/licenses/by/4.0/. The Creative Commons Public Domain Dedication waiver (http://creativecommons.org/publicdomain/ zero/1.0/) applies to the data made available in this article, unless otherwise stated in a credit line to the data. 
Definition 2.1 [15] A $b$-metric is a mapping $\partial: \mathscr{E} \times \mathscr{E} \rightarrow[0,+\infty)$ that satisfies the properties below for all $\varepsilon, \wp, \zeta$ in $\mathscr{E}$ and some $\mathrm{s} \geq 1$,
(a) $\partial(\varepsilon, \wp)=0$ if and if $\varepsilon=\wp$,
(b) $\partial(\varepsilon, \wp)=\partial(\wp, \varepsilon)$,
(c) $\partial(\varepsilon, \wp) \leq s(\partial(\varepsilon, \zeta)+\partial(\zeta, \wp))$.

A $b$-metric space is specified as $(\mathscr{E}, \check{\partial}, \mathrm{s})$.

Example 2.2 The space $L_{q}[0,1]$, where $0<q<1$ of all real functions $f(t), t \in[0,1]$ such that $\int_{0}^{1}|f(t)|^{q} d t<\infty$ is a $b$-metric space if we take $ð(\varepsilon, \wp)=\int_{0}^{1}\left(|f(t)-g(t)|^{q} d t\right)^{\frac{1}{q}}$, for all $\varepsilon, \wp \in L_{q}[0,1]$.

Note 2.3 Every metric space is a $b$-metric space with $\mathrm{s}=1$, but in general a $b$-metric space need not necessarily be a metric space, as in below example 2.4 is $b$-metric space but not a metric space. Thus, the class of $b$-metric spaces is larger than the class of metric spaces.

Example 2.4 Let $\mathscr{E}=\mathbb{R}$ and define the mapping $\partial: \mathscr{E} \times \mathscr{E} \rightarrow \mathbb{R}^{+}$by $\partial(\varepsilon, \wp)=|\varepsilon-\wp|^{2}$, for all $\varepsilon, \wp \in \mathscr{E}$. Then $(\mathscr{E}, ð)$ is a $b$-metric space with coefficient $\mathrm{s}=2$.

The generalization of the above Example 2.4 is as follows:

Example 2.5 Let $(\mathscr{E}, d)$ be a metric space and $q \geq 1$ be a given real number. Then $\partial(\varepsilon, \wp)=[d(\varepsilon, \wp)]^{q}$ is a $b$-metric on $\mathscr{E}$ with parameter $\mathrm{s} \leq 2^{\mathrm{q}-1}$.

Definition 2.6 $[10,15]$ In a $b$-metric space,

(1) if $\partial\left(\varepsilon_{n}, \varepsilon\right) \rightarrow 0$ as $n \rightarrow+\infty$ then $\left\{\varepsilon_{n}\right\}$ is said to be convergent to $\varepsilon$.

(2) if $\partial\left(\varepsilon_{n}, \varepsilon_{m}\right) \rightarrow 0$ as $n, m \rightarrow+\infty$ then $\left\{\varepsilon_{n}\right\}$ is a Cauchy sequence.

(3) if $(\mathscr{E}, ð, \mathrm{~s})$ is a complete $b$-metric space then very Cauchy sequence is convergent.

Definition 2.7 $\quad[15,33]$ If $\mathscr{E}$ is a partial ordered set with respect to an ordered relation $\preceq$ and $\partial$ is a metric on it, then $(\mathscr{E}, \partial, \preceq)$ is a partially ordered metric space. $(\mathscr{E}, ð, \preceq)$ is complete partially ordered $b$-metric space, despite the fact that $\partial$ is complete.
Definition 2.8 [33] Let $h: \mathscr{E} \rightarrow \mathscr{E}$ be a mapping. If $h(\varepsilon) \preceq h(\wp)$ for all $\varepsilon, \wp \in \mathscr{E}$ with $\varepsilon \preceq \wp$, then $h$ is called monotone nondecreasing mapping.

Definition 2.9 [12] Let $h, \mathscr{I}: \mathscr{A} \rightarrow \mathscr{A}$ be two mappings, and $\mathscr{A} \neq \emptyset \subseteq \mathscr{E}$. If $h \varepsilon=\mathscr{I} \varepsilon=\varepsilon(h \varepsilon=\mathscr{I} \varepsilon)$ for $\varepsilon \in \mathscr{A}$, then $\varepsilon$ is called a common fixed point (coincidence point) of $h$ and $\mathscr{I}$.

Definition 2.10 [12] If $h \mathscr{I} \varepsilon=\mathscr{I} h \varepsilon$ for all $\varepsilon \in \mathscr{A}$, then $h$ and $\mathscr{I}$ are commuting.

Definition 2.11 $[12,33]$ The two self mappings $h$ and $\mathscr{I}$ are known to be compatible, if $\lim d\left(\mathscr{I} h \varepsilon_{n}, h \mathscr{I} \varepsilon_{n}\right)=0$ for every sequence $\left\{\varepsilon_{n}\right\}$ in $\mathscr{E}$ such that $\lim _{n \rightarrow+\infty} h \varepsilon_{n}=\lim _{n \rightarrow+\infty} \mathscr{I}_{\xi}=\mu$, for some $\mu \in \mathscr{A}$.

Definition $2.12[12,33]$ If $h \varepsilon=\mathscr{I} \varepsilon$ for some $\varepsilon \in \mathscr{A}$, then $h \mathscr{I}_{\varepsilon}=\mathscr{I} h \varepsilon$, the mappings $h$ and $\mathscr{I}$ are called weakly compatible.

Definition 2.13 [33] If $h \varepsilon \preceq h \wp$ implies $\mathscr{I} \varepsilon \preceq \mathscr{I} \wp$ for each $\varepsilon, \wp \in \mathscr{E}$, then the mapping $\mathscr{I}$ is called monotone $h$ -nondecreasing.

Definition 2.14 [11] Let $\mathscr{I}: \mathscr{E} \times \mathscr{E} \rightarrow \mathscr{E} \quad$ and $h: \mathscr{E} \rightarrow \mathscr{E}$ are two mappings,

(a) a point $(\varepsilon, \wp) \in \mathscr{E} \times \mathscr{E}$ is coupled coincidence point of $\mathscr{I}$ and $h$, if $\mathscr{I}(\varepsilon, \wp)=h \varepsilon$ and $\mathscr{I}(\wp, \varepsilon)=h \wp$. In particular, if $h$ is an identity mapping, then $(\varepsilon, \wp)$ is a coupled fixed point of $\mathscr{I}$.

(b) a point $\varepsilon \in \mathscr{E}$ is a common fixed point of $\mathscr{I}$ and $h$, if $\mathscr{I}(\varepsilon, \varepsilon)=h \varepsilon=\varepsilon$.

(c) if $\mathscr{I}(h \varepsilon, h \wp)=h(\mathscr{I} \varepsilon, \mathscr{I} \wp)$ for all $\varepsilon, \wp \in \mathscr{E}$, then $\mathscr{I}$ and $h$ are commuting each other.

(d) If every two elements of $\mathscr{A} \subseteq \mathscr{E}$ are comparable, then the set $\mathscr{A}$ is called a well ordered set.

Definition 2.15 A self mapping $\breve{\psi}$ on $[0,+\infty)$ that meets the conditions below is known as an altering distance function:

(a) $\check{\psi}$ is a non-decreasing and continuous function,

(b) $\breve{\psi}(\ell)=0$ if and only if $\ell=0$.

As seen above, the symbol $\hat{\Phi}$ represents the set of all altering distance functions. 
Similarly, $\quad \hat{\Psi}:\{\hat{\eta} \mid \hat{\eta}$ is a lower semi-continuous self mapping on $[0,+\infty)$ and, $\hat{\eta}(\ell)=0$ if and only if $\ell=0\}$.

The presented lemmas under here are frequently used in our main results.

Lemma 2.16 [27] Let $h: \mathscr{E} \rightarrow \mathscr{E}$ be a mapping, and $\mathscr{E} \neq \emptyset$. Then $\mathscr{M} \subseteq \mathscr{E}$ occurs, resulting in $h \mathscr{M}=h \mathscr{E}$, where $h: \mathscr{M} \rightarrow \mathscr{E}$ is one-to-one.

Lemma 2.17 [4] Let $\left\{\varepsilon_{n}\right\}$ and $\left\{\wp_{n}\right\}$ be two sequences and $b$-convergent to $\varepsilon$ and $\wp$ in a $b$-metric space $(\mathscr{E}, \partial, \mathrm{s}, \preceq)$, where $\mathrm{s}>1$. Then

$$
\begin{aligned}
\frac{1}{\mathrm{~s}^{2}} \partial(\varepsilon, \wp) & \leq \lim _{n \rightarrow+\infty} \inf \partial\left(\varepsilon_{n}, \wp_{n}\right) \\
& \leq \lim _{n \rightarrow+\infty} \sup \partial\left(\varepsilon_{n}, \wp_{n}\right) \leq \mathrm{s}^{2} \partial(\varepsilon, \wp) .
\end{aligned}
$$

In particular, if $\varepsilon=\wp$, then $\lim _{n \rightarrow+\infty} \partial\left(\varepsilon_{n}, \wp_{n}\right)=0$. In addition, for every $\tau \in \mathscr{E}$, we get

$$
\frac{1}{\mathrm{~S}} \partial(\varepsilon, \tau) \leq \lim _{n \rightarrow+\infty} \inf \partial\left(\varepsilon_{n}, \tau\right) \leq \lim _{n \rightarrow+\infty} \sup \partial\left(\varepsilon_{n}, \tau\right) \leq \operatorname{sd}(\varepsilon, \tau) .
$$

$\left\{\varepsilon_{n}\right\} \subset \mathscr{E}$ by $\varepsilon_{n+1}=\mathscr{I} \varepsilon_{n}$ for all $n \geq 0$. However, we can deduce the following as $\mathscr{I}$ is nondecreasing,

$$
\begin{aligned}
\varepsilon_{0} \prec \mathscr{I} \varepsilon_{0} & =\varepsilon_{1} \preceq \mathscr{I} \varepsilon_{1}=\varepsilon_{2} \preceq \ldots \preceq \mathscr{I} \varepsilon_{n-1} \\
& =\varepsilon_{n} \preceq \mathscr{I} \varepsilon_{n}=\varepsilon_{n+1} \preceq \ldots \ldots .
\end{aligned}
$$

If $\varepsilon_{n_{0}}=\varepsilon_{n_{0}+1}$ for $n_{0} \in \mathbb{N}$, then $\varepsilon_{n_{0}}$ is a fixed point of $\mathscr{I}$ from (3). Otherwise, for all $n \geq 1, \varepsilon_{n} \neq \varepsilon_{n+1}$. For $n \geq 1$, let $D_{n}=\partial\left(\varepsilon_{n+1}, \varepsilon_{n}\right)$. We know that for every $n \geq 1$, $\varepsilon_{n-1} \prec \varepsilon_{n}$ and, then the equation (1) becomes

$$
\begin{aligned}
\check{\psi}\left(D_{n}\right)=\check{\psi}\left(\check{\partial}\left(\varepsilon_{n}, \varepsilon_{n+1}\right)\right) & =\check{\psi}\left(ð\left(\mathscr{I} \varepsilon_{n-1}, \mathscr{I} \varepsilon_{n}\right)\right) \\
& \leq \check{\psi}\left(\operatorname{s} \partial\left(\mathscr{I} \varepsilon_{\mathrm{n}-1}, \mathscr{I} \varepsilon_{\mathrm{n}}\right)\right) \\
& \leq \check{\psi}\left(\mathscr{P}\left(\varepsilon_{n-1}, \varepsilon_{n}\right)\right)-\hat{\eta}\left(\mathscr{P}\left(\varepsilon_{n-1}, \varepsilon_{n}\right)\right)
\end{aligned}
$$

From (4), we get

$$
\partial\left(\varepsilon_{n}, \varepsilon_{n+1}\right)=\partial\left(\mathscr{I} \varepsilon_{n-1}, \mathscr{I} \varepsilon_{n}\right) \leq \frac{1}{\jmath} \mathscr{P}\left(\varepsilon_{n-1}, \varepsilon_{n}\right),
$$

where

$$
\begin{aligned}
\mathscr{P}\left(\varepsilon_{n-1}, \varepsilon_{n}\right)= & \max \left\{\frac{\partial\left(\varepsilon_{n}, \mathscr{I} \varepsilon_{n}\right)\left[1+\partial\left(\varepsilon_{n-1}, \mathscr{I} \varepsilon_{n-1}\right)\right]}{1+\partial\left(\varepsilon_{n-1}, \varepsilon_{n}\right)}, \frac{\partial\left(\varepsilon_{n-1}, \mathscr{I} \varepsilon_{n}\right)+\partial\left(\varepsilon_{n}, \mathscr{I} \varepsilon_{n-1}\right)}{2 s}, \partial\left(\varepsilon_{n-1}, \mathscr{I} \varepsilon_{n-1}\right), \partial\left(\varepsilon_{n}, \mathscr{I} \varepsilon_{n}\right), \partial\left(\varepsilon_{n-1}, \varepsilon_{n}\right)\right\}(6) \\
& \leqslant \max \left\{\partial\left(\varepsilon_{n}, \varepsilon_{n+1}\right), \frac{\partial\left(\varepsilon_{n-1}, \varepsilon_{n}\right)+\partial\left(\varepsilon_{n}, \varepsilon_{n+1}\right)}{2}, \partial\left(\varepsilon_{n-1}, \varepsilon_{n}\right)\right\} \leqslant \max \left\{D_{n}, D_{n-1}\right\} .
\end{aligned}
$$

\section{Main results}

We start this section with the following fixed point theorem in an ordered $b$-metric space.

Theorem 3.1 Suppose $(\mathscr{E}, \partial \mathrm{d}, \mathrm{s}, \preceq)$ is a complete partially ordered b-metric space with $\mathrm{s}>1$. A mapping $\mathscr{I}: \mathscr{E} \rightarrow \mathscr{E}$ is continuous and nondecreasing with respect to $\preceq$. If $\varepsilon_{0} \in \mathscr{E}$ is such that $\varepsilon_{0} \preceq \mathscr{I} \varepsilon_{0}$ and the following contraction condition is fulfilled, then $\mathscr{I}$ has a fixed point in $\mathscr{E}$.

$$
\breve{\psi}(\mathrm{s} \partial(\mathscr{I} \varepsilon, \mathscr{I} \wp)) \leq \breve{\psi}(\mathscr{P}(\varepsilon, \wp))-\hat{\eta}(\mathscr{P}(\varepsilon, \wp)),
$$

for $\check{\psi} \in \hat{\Phi}, \hat{\eta} \in \hat{\Psi}$ and for any $\varepsilon, \wp \in \mathscr{E}$ such that $\varepsilon \preceq \wp$ and where
If $\max \left\{D_{n}, D_{n-1}\right\}=D_{n}$ for certain $n \geq 1$, equation (5) is then accompanied by

$$
\partial\left(\varepsilon_{n}, \varepsilon_{n+1}\right) \leq \frac{1}{\sigma} \partial\left(\varepsilon_{n}, \varepsilon_{n+1}\right),
$$

this is a contradiction. Thus, $\max \left\{D_{n}, D_{n-1}\right\}=D_{n-1}$ for $n \geq 1$. Hence, equation (5) becomes

$$
ð\left(\varepsilon_{n}, \varepsilon_{n+1}\right) \leq \frac{1}{\jmath} \partial\left(\varepsilon_{n}, \varepsilon_{n-1}\right) .
$$

Since $\frac{1}{j} \in(0,1)$, then $\left\{\varepsilon_{n}\right\}$ is a Cauchy sequence from $[1,6,8,18]$. Also, the completeness of $\mathscr{E}$ gives that $\varepsilon_{n} \rightarrow \mu \in \mathscr{E}$.

$$
\mathscr{P}(\varepsilon, \wp)=\max \left\{\frac{\partial(\wp, \mathscr{I} \wp)[1+\partial(\varepsilon, \mathscr{I} \varepsilon)]}{1+\partial(\varepsilon, \wp)}, \frac{\partial(\varepsilon, \mathscr{I} \wp)+\partial(\wp, \mathscr{I} \varepsilon)}{2 s}, \partial(\varepsilon, \mathscr{I} \varepsilon), \partial(\wp, \mathscr{I} \wp), \partial(\varepsilon, \wp)\right\} .
$$

Proof For some $\varepsilon_{0} \in \mathscr{E}$ with $\mathscr{I} \varepsilon_{0}=\varepsilon_{0}$, then the result is trivial. Assuming that $\varepsilon_{0} \prec \mathscr{I} \varepsilon_{0}$, we describe a sequence
We may also deduce the following from the continuity of $\mathscr{I}$ 


$$
\mathscr{I} \mu=\mathscr{I}\left(\lim _{n \rightarrow+\infty} \varepsilon_{n}\right)=\lim _{n \rightarrow+\infty} \mathscr{I} \varepsilon_{n}=\lim _{n \rightarrow+\infty} \varepsilon_{n+1}=\mu .
$$

As a result, $\mathscr{I}$ in $\mathscr{E}$ has a fixed point $\mu$.

The continuity assumption on $\mathscr{I}$ is extracted from Theorem 3.1 and used to derive the following theorem.

Theorem 3.2 In Theorem 3.1, if $\mathscr{E}$ satisfies below condition, then $\mathscr{I}$ has a fixed point.

If a non-decreasing sequence $\left\{\varepsilon_{n}\right\}$

$\subseteq \mathscr{E}$ and $\varepsilon_{n} \rightarrow \sigma$ then $\varepsilon_{n} \leq \sigma$,

for each $n \in \mathbb{N}$, i.e., $\sigma=\sup \varepsilon_{n}$.

Proof We have an increasing sequence $\left\{\varepsilon_{n}\right\} \subseteq \mathscr{E}$ that eventually converges to some $\sigma \in \mathscr{E}$ as a result of Theorem 3.1. But by the hypotheses for all $n, \varepsilon_{n} \preceq \sigma$, which means that $\sigma=\sup \varepsilon_{n}$.
In the above theorems, the fixed point is unique if $\mathscr{E}$ meets the following condition.

There exists a $\sigma$ in $\mathscr{E}$ that is comparable to $\varepsilon$ and $\wp$, for each $\varepsilon, \wp \in \mathscr{E}$.

Theorem 3.3 If $\mathscr{E}$ assumes the condition (13) in Theorem $3.1 \mathcal{E} 3.2$, then $\mathscr{I}$ has a unique fixed point in $\mathscr{E}$.

Proof Theorems $3.1 \& 3.2$ show that the set of fixed points of $\mathscr{I}$ is nonempty. Assume $\varepsilon^{*} \neq \wp^{*}$ are fixed points of $\mathscr{I}$ to ensure uniqueness. Following that,

$$
\begin{aligned}
\check{\psi}\left(\check{(}\left(\mathscr{I} \varepsilon^{*}, \mathscr{I} \wp^{*}\right)\right) & \leq \check{\psi}\left(s ð\left(\mathscr{I} \varepsilon^{*}, \mathscr{I} \wp^{*}\right)\right) \\
& \leq \check{\psi}\left(\mathscr{P}\left(\varepsilon^{*}, \wp^{*}\right)\right)-\hat{\eta}\left(\mathscr{P}\left(\varepsilon^{*}, \wp^{*}\right)\right),
\end{aligned}
$$

where

We can now assert that $\sigma$ is a fixed point of $\mathscr{I}$. Assume that $\mathscr{I} \sigma \neq \sigma$. Let

$$
\mathscr{P}\left(\varepsilon^{*}, \wp^{*}\right)=\max \left\{\frac{\partial\left(\wp^{*}, \mathscr{I} \wp^{*}\right)\left[1+\partial\left(\varepsilon^{*}, \mathscr{I} \varepsilon^{*}\right)\right]}{1+\partial\left(\varepsilon^{*}, \wp^{*}\right)}, \frac{\partial\left(\varepsilon^{*}, \mathscr{I} \wp^{*}\right)+\partial\left(\wp^{*}, \mathscr{I} \varepsilon^{*}\right)}{2 s}, \partial\left(\varepsilon^{*}, \mathscr{I} \varepsilon^{*}\right), \partial\left(\wp^{*}, \mathscr{I} \wp^{*}\right), \partial\left(\varepsilon^{*}, \wp^{*}\right)\right\} .
$$

$$
\mathscr{P}\left(\varepsilon_{n}, \sigma\right)=\max \left\{\frac{\partial(\sigma, \mathscr{I} \sigma)\left[1+\partial\left(\varepsilon_{n}, \mathscr{I} \varepsilon_{n}\right)\right]}{1+\partial\left(\varepsilon_{n}, \sigma\right)}, \frac{\partial\left(\varepsilon_{n}, \mathscr{I} \sigma\right)+\partial\left(\sigma, \mathscr{I} \varepsilon_{n}\right)}{2 s}, \partial\left(\varepsilon_{n}, \mathscr{I} \varepsilon_{n}\right), \partial(\sigma, \mathscr{I} \sigma), \partial\left(\varepsilon_{n}, \sigma\right)\right\}
$$

then taking limit as $n \rightarrow+\infty$ in the equation (9) and making use of $\lim _{n \rightarrow+\infty} \varepsilon_{n}=\sigma$, we get

$$
\lim _{n \rightarrow+\infty} \mathscr{P}\left(\varepsilon_{n}, \sigma\right)=\max \{\partial(\sigma, \mathscr{I} \sigma), 0\}=ð(\sigma, \mathscr{I} \sigma) .
$$

Since, $\varepsilon_{n} \preceq \sigma$ for each $n$, then we obtain the following from equations (1) and (9)

$$
\begin{aligned}
\check{\psi}\left(\partial\left(\varepsilon_{n+1}, \mathscr{I} \sigma\right)\right) & =\check{\psi}\left(ð\left(\mathscr{I} \varepsilon_{n}, \mathscr{I} \sigma\right)\right) \leq \breve{\psi}\left(s ð\left(\mathscr{I} \varepsilon_{n}, \mathscr{I} \sigma\right)\right) \\
& \leq \check{\psi}\left(\mathscr{P}\left(\varepsilon_{n}, \sigma\right)\right)-\hat{\eta}\left(\mathscr{P}\left(\varepsilon_{n}, \sigma\right)\right) .
\end{aligned}
$$

Take limit as $n \rightarrow+\infty$ in (11) and from equation (10) as well as the properties of $\breve{\psi}, \hat{\eta}$, we have

$$
\check{\psi}(\partial(\sigma, \mathscr{I} \sigma)) \leq \check{\psi}(\partial(\sigma, \mathscr{I} \sigma))-\hat{\eta}(\partial(\sigma, \mathscr{I} \sigma))<\breve{\psi}(\partial(\sigma, \mathscr{I} \sigma)) .
$$

This is a contradiction to $\mathscr{I} \sigma \neq \sigma$. Hence, $\mathscr{I} \sigma=\sigma$.
Therefore from equations (14) and (15), we have

$$
\begin{aligned}
\check{\psi}\left(\partial\left(\varepsilon^{*}, \wp^{*}\right)\right)= & \check{\psi}\left(\delta\left(\mathscr{I} \varepsilon^{*}, \mathscr{I} \wp^{*}\right)\right) \leq \check{\psi}\left(\partial\left(\varepsilon^{*}, \wp^{*}\right)\right) \\
& -\hat{\eta}\left(ð\left(\varepsilon^{*}, \wp^{*}\right)\right)<\check{\psi}\left(ð\left(\varepsilon^{*}, \wp^{*}\right)\right),
\end{aligned}
$$

this contradicts to $\varepsilon^{*} \neq \wp^{*}$. Hence, $\varepsilon^{*}=\wp^{*}$.

Now, we have the below corollary from Theorems 3.1 to 3.3 .

Corollary 3.4 Let $(\mathscr{E}, ð, \preceq)$ be a partially ordered $b$-metric space. Suppose the mappings $\mathscr{I}, h: \mathscr{E} \rightarrow \mathscr{E}$ are continuous such that

$\left(C_{1}\right)$. 


$$
\check{\psi}(\mathrm{s} \check{\partial}(\mathscr{I} \varepsilon, \mathscr{I} \wp)) \leq \check{\psi}\left(\mathscr{P}_{h}(\varepsilon, \wp)\right)-\hat{\eta}\left(\mathscr{P}_{h}(\varepsilon, \wp)\right),
$$

for every $\varepsilon, \wp \in \mathscr{E}$ with $h \varepsilon \preceq h \wp, \mathrm{s}>1, \check{\psi} \in \hat{\Phi}, \hat{\eta} \in \hat{\Psi}$ and, where

$$
\begin{aligned}
\partial\left(\mathscr{I}_{v}, h v\right) \leq & s \partial\left(\mathscr{I}_{v}, \mathscr{I}\left(h \varepsilon_{n}\right)\right)+s^{2} \partial\left(\mathscr{I}\left(h \varepsilon_{n}\right), h\left(\mathscr{I} \varepsilon_{n}\right)\right) \\
& +s^{2} \partial\left(h\left(\mathscr{I} \varepsilon_{n}\right), h v\right) .
\end{aligned}
$$

$$
\mathscr{P}_{h}(\varepsilon, \wp)=\max \left\{\frac{\partial(h \wp, \mathscr{I} \wp)[1+\partial(h \varepsilon, \mathscr{I} \varepsilon)]}{1+\partial(h \varepsilon, h \wp)}, \frac{\partial(h \varepsilon, \mathscr{I} \wp)+\partial(h \wp, \mathscr{I} \varepsilon)}{2 s}, \partial(h \varepsilon, \mathscr{I} \varepsilon), \partial(h \wp, \mathscr{I} \wp), \partial(h \varepsilon, h \wp)\right\} .
$$

$\left(C_{2}\right) . \quad \mathscr{I} \mathscr{E} \subset h \mathscr{E}$ and $h \mathscr{E} \subseteq \mathscr{E}$ is complete,

$\left(C_{3}\right) . \quad \mathscr{I}$ is monotone $h$-non-decreasing and

$\left(C_{4}\right) . \quad \mathscr{I}$ and $h$ are compatible.

If for some $\varepsilon_{0} \in \mathscr{E}$ such that $h \varepsilon_{0} \preceq \mathscr{I} \varepsilon_{0}$, then a pair of mappings $(\mathscr{I}, h)$ has a coincidence point in $\mathscr{E}$.

Proof By Lemma 2.16, there exists $\mathscr{M} \subset \mathscr{E}$ such that $h \mathscr{M}=h \mathscr{E}$ and $h: \mathscr{M} \rightarrow \mathscr{E}$ is one-to-one. Now define a $\operatorname{map} k: h \mathscr{M} \rightarrow h \mathscr{M}$ by $k(h \varepsilon)=\mathscr{I} \varepsilon, \varepsilon \in \mathscr{M}$. Since $h$ is one-to-one on $\mathscr{M}, k$ is well defined. Then, $h \mathscr{M}=h \mathscr{E}$ is complete and then (17) becomes

$$
\check{\psi}(s ð(k(h \varepsilon), k(h \wp))) \leq \check{\psi}\left(\mathscr{P}_{h}(\varepsilon, \wp)\right)-\hat{\eta}\left(\mathscr{P}_{h}(\varepsilon, \wp)\right),
$$

for every $\varepsilon, \wp \in \mathscr{E}$ with $h \varepsilon \preceq h \wp$ and, where
Taking $n \rightarrow+\infty$ in (22) and the continuity of $\mathscr{I}, h$ and (21), we get $\partial\left(\mathscr{I}_{v}, h v\right)=0$. That is $\mathscr{I}_{v}=h v$. Therefore, $v$ is a coincidence point of $\mathscr{I}, h$.

The following result can get from Corollary 3.4 by weakening its hypotheses.

Corollary 3.5 If $\mathscr{E}$ satisfies the following condition in Corollary 3.4,

for very nondecreasing sequence $\left\{h \varepsilon_{n}\right\}$

$\subseteq \mathscr{E}$ such that $h \varepsilon_{n} \rightarrow h \sigma$, then

$h \varepsilon_{n} \leq h \sigma(n \geq 0)$, i.e., $h \sigma=\sup h \varepsilon_{n}$.

then, if $h \mu \preceq h(h \mu)$ for some coincidence point $\mu$, a coincidence point exists for the weakly compatible mappings $(\mathscr{I}, h)$. Moreover, $(\mathscr{I}, h)$ has only one common fixed

$$
\begin{aligned}
\mathscr{P}_{h}(\varepsilon, \wp)= & \max \left\{\frac{\partial(h \wp, k \partial(h \wp))[1+\partial(h \varepsilon, k \partial(h \varepsilon))]}{1+\partial(h \varepsilon, h \wp)}, \frac{\partial(h \varepsilon, k \partial(h \wp))+\partial(h \wp, k \partial(h \varepsilon))}{2 s},\right. \\
& \partial(h \varepsilon, k \partial(h \varepsilon)), \partial(h \wp, k \partial(h \wp)), \partial(h \varepsilon, h \wp)\} .
\end{aligned}
$$

Let $\varepsilon_{0} \in \mathscr{M}$ such that $h \varepsilon_{0} \preceq \mathscr{I} \varepsilon_{0}=k\left(h \varepsilon_{0}\right)$. Choose $\varepsilon_{1} \in \mathscr{M}$ such that $h \varepsilon_{1}=\mathscr{I} \varepsilon_{0}=k\left(h \varepsilon_{0}\right)$. By continuing this process, we obtain a sequence $\left\{h \varepsilon_{n}\right\} \subset h \mathscr{M}$ such that $h \varepsilon_{n+1}=\mathscr{I} \varepsilon_{n}=k\left(h \varepsilon_{n}\right)$ for $n \geq 0$. By using the similar argument as in the proof of Theorem 3.1, we obtain that $\left\{h \varepsilon_{n}\right\} \subset h \mathscr{M}$ is a $b$-Cauchy sequence. Since $h \mathscr{M}$ is complete, there exists $v \in h \mathscr{M}$ such that $\lim _{n \rightarrow+\infty} h \varepsilon_{n}=v \in h \mathscr{E}$. Then
$\lim _{n \rightarrow+\infty} h \varepsilon_{n}=\lim _{n \rightarrow+\infty} \mathscr{I} \varepsilon_{n-1}=v$.

From the condition $\left(C_{4}\right)$, we have

$$
\lim _{n \rightarrow+\infty} \partial\left(h\left(\mathscr{I} \varepsilon_{n}\right), \mathscr{I}\left(h \varepsilon_{n}\right)\right)=0 .
$$

Furthermore, the triangular inequality of $b$-metric, we have point if and only if the set of common fixed points is well ordered.

Proof A pair of mappings $(\mathscr{I}, h)$ has a coincidence point, according to Theorem 3.3 and Corollary 3.4.

Next, assume that a pair of mappings $(\mathscr{I}, h)$ is weakly compatible. Let $v \in \mathscr{E}$ be a point with $v=\mathscr{I} \mu=h \mu$. Then, $\mathscr{I} v=\mathscr{I}(h \mu)=h(\mathscr{I} \mu)=h v$.

Therefore, 


$$
\begin{aligned}
\mathscr{P}_{h}(\mu, v) & =\max \left\{\frac{\partial(h v, I v)[1+\partial(h \mu, I \mu)]}{1+\partial(h \mu, h v)}, \frac{\partial(h \mu, I v)+\partial(h v, I \mu)}{2 s}, \partial(h \mu, I \mu), \partial(h v, I v), \partial(h \mu, h v)\right\} \\
& =\max \left\{0, \frac{\partial(I \mu, I v)}{s}, \partial(I \mu, I v)\right\}=\partial(I \mu, I v) .
\end{aligned}
$$

Thus from equation (17), we get

$$
\begin{aligned}
\check{\psi}\left(ð\left(\mathscr{I} \mu, \mathscr{I}_{v}\right)\right) & \leq \check{\psi}\left(\mathscr{P}_{h}(\mu, v)\right)-\hat{\eta}\left(\mathscr{P}_{h}(\mu, v)\right) \\
& \leq \check{\psi}\left(ð\left(\mathscr{I} \mu, \mathscr{I}_{v}\right)\right)-\hat{\eta}\left(ð\left(\mathscr{I} \mu, \mathscr{I}_{v}\right)\right) .
\end{aligned}
$$

By the property of $\hat{\eta}$, we get $\precsim(\mathscr{I} \mu, \mathscr{I} v)=0$ implies that $\mathscr{I}_{v}=h v=v$.

Finally, we can deduce from Theorem 3.3 that $(\mathscr{I}, h)$ has only one common fixed point if and only if the common fixed points of $(\mathscr{I}, h)$ is well ordered.

Remark 3.6 Theorems 3.1 to 3.3 are respectively the extension of Theorems 2.1,.2.2 \& 2.3 of [27].

Remark 3.7 Corollaries $3.4 \& 3.5$ are the generalizations of Corollaries $2.1 \& 2.2$ of [12] respectively.

Definition 3.8 Consider a partially ordered $b$-metric space, $(\mathscr{E}, ð, \preceq)$. A mapping $\mathscr{I}: \mathscr{E} \times \mathscr{E} \rightarrow \mathscr{E}$ is known to be a generalized $(\check{\psi}, \hat{\eta})$-contractive mapping with regards to $h: \mathscr{E} \rightarrow \mathscr{E}$, if

$\check{\psi}\left(s^{k} \partial(\mathscr{I}(\varepsilon, \wp), \mathscr{I}(\zeta, \mathfrak{I}))\right) \leq \check{\psi}\left(\mathscr{P}_{h}(\varepsilon, \wp, \zeta, \mathfrak{I})\right)-\hat{\eta}\left(\mathscr{P}_{h}(\varepsilon, \wp, \zeta, \mathfrak{I})\right)$,

for all $\varepsilon, \wp, \zeta, \mathfrak{I} \in \mathscr{E}$ with $h \varepsilon \preceq h \zeta$ and $h \wp \succeq h \mathfrak{I}, k>2$, $s>1, \breve{\psi} \in \hat{\Phi}, \hat{\eta} \in \hat{\Psi}$ and where with $h$. Assume that, if for some $\left(\varepsilon_{0}, \wp_{0}\right) \in \mathscr{E} \times \mathscr{E}$ such that $\quad h \varepsilon_{0} \preceq \mathscr{I}\left(\varepsilon_{0}, \wp_{0}\right), \quad h \wp_{0} \succeq \mathscr{I}\left(\wp_{0}, \varepsilon_{0}\right) \quad$ and $\mathscr{I}(\mathscr{E} \times \mathscr{E}) \subseteq h(\mathscr{E})$, then $\mathscr{I}$ and $h$ have a coupled coincidence point in $\mathscr{E}$.

Proof From Theorem 2.2 of [7], there exist two sequences $\left\{\varepsilon_{n}\right\}$ and $\left\{\wp_{n}\right\}$ in $\mathscr{E}$ such that

$$
h \varepsilon_{n+1}=\mathscr{I}\left(\varepsilon_{n}, \wp_{n}\right), \quad h \wp_{n+1}=\mathscr{I}\left(\wp_{n}, \varepsilon_{n}\right), n \geq 0 .
$$

In particular, the sequences $\left\{h \varepsilon_{n}\right\}$ and $\left\{h \wp_{n}\right\}$ are non-decreasing and non-increasing in $\mathscr{E}$. Put $\varepsilon=\varepsilon_{n}, \wp=\wp_{n}, \zeta=\varepsilon_{n+1}, \mathfrak{I}=\wp_{n+1}$ in (26), we get

$$
\begin{aligned}
\check{\psi}\left(s^{k} \partial\left(h \varepsilon_{n+1}, h \varepsilon_{n+2}\right)\right)= & \check{\psi}\left(s^{k} \partial\left(\mathscr{I}\left(\varepsilon_{n}, \wp_{n}\right), \mathscr{I}\left(\varepsilon_{n+1}, \wp_{n+1}\right)\right)\right) \\
\leq & \check{\psi}\left(\mathscr{P}_{h}\left(\varepsilon_{n}, \wp_{n}, \varepsilon_{n+1}, \wp_{n+1}\right)\right) \\
& -\hat{\eta}\left(\mathscr{P}_{h}\left(\varepsilon_{n}, \wp_{n}, \varepsilon_{n+1}, \wp_{n+1}\right)\right),
\end{aligned}
$$

where

$$
\mathscr{P}_{h}\left(\varepsilon_{n}, \wp_{n}, \varepsilon_{n+1}, \wp_{n+1}\right) \leq \max \left\{\partial\left(h \varepsilon_{n}, h \varepsilon_{n+1}\right), \partial\left(h \varepsilon_{n+1}, h \varepsilon_{n+2}\right)\right\} .
$$

Therefore from (27), we have

$$
\begin{aligned}
\check{\psi}\left(s^{k} \partial\left(h \varepsilon_{n+1}, h \varepsilon_{n+2}\right)\right) \leq & \check{\psi}\left(\max \left\{\partial\left(h \varepsilon_{n}, h \varepsilon_{n+1}\right), \partial\left(h \varepsilon_{n+1}, h \varepsilon_{n+2}\right)\right\}\right) \\
& -\hat{\eta}\left(\max \left\{\partial\left(h \varepsilon_{n}, h \varepsilon_{n+1}\right), \partial\left(h \varepsilon_{n+1}, h \varepsilon_{n+2}\right)\right\}\right) .
\end{aligned}
$$

Similarly by taking $\varepsilon=\wp_{n+1}, \wp=\varepsilon_{n+1}, \zeta=\varepsilon_{n}, \mathfrak{I}=\varepsilon_{n}$ in (26), we get

$$
\begin{aligned}
& \check{\psi}\left(s^{k} \partial\left(h \wp_{n+1}, h \wp_{n+2}\right)\right) \leq \check{\psi}\left(\max \left\{\partial\left(h \wp_{n}, h \wp_{n+1}\right), \partial\left(h \wp_{n+1}, h \wp_{n+2}\right)\right\}\right) \\
& -\hat{\eta}\left(\max \left\{\partial\left(h \wp_{n}, h \wp_{n+1}\right), \partial\left(h \wp_{n+1}, h \wp_{n+2}\right)\right\}\right) . \\
& \mathscr{P}_{h}(\varepsilon, \wp, \zeta, \Im)=\max \left\{\frac{\partial(h \zeta, \mathscr{I} \partial(\zeta, \Im))[1+\partial(h \varepsilon, \overline{\mathscr{I} \partial(\varepsilon, \wp))]}}{1+\partial(h \varepsilon, h \zeta)}, \frac{\partial(h \varepsilon, \mathscr{I} \partial(\zeta, \Im))+\partial(h \zeta, \mathscr{I} \partial(\varepsilon, \wp))}{2 s},\right. \\
& \partial(h \varepsilon, \mathscr{I} \partial(\varepsilon, \wp)), \partial(h \zeta, \mathscr{I} \partial(\zeta, \Im)), \partial(h \varepsilon, h \zeta)\}
\end{aligned}
$$

Theorem 3.9 Suppose that $(\mathscr{E}, \check{\partial}, \preceq)$ is a complete partially ordered b-metric space. A mapping $\mathscr{I}: \mathscr{E} \times \mathscr{E} \rightarrow \mathscr{E}$ satisfies the condition (26) and $\mathscr{I}, h$ are continuous, $\mathscr{I}$ has mixed h-monotone property and also commutes
We know that $\max \left\{\check{\psi}\left(l_{1}\right), \check{\psi}\left(l_{2}\right)\right\}=\check{\psi}\left\{\max \left\{l_{1}, l_{2}\right\}\right\}$ for $l_{1}, l_{2} \in[0,+\infty)$. Then by adding (29) and (30) together we get,

$$
\begin{aligned}
\check{\psi}\left(s^{k} \Gamma_{n}\right) & \leq \check{\psi}\left(\max \left\{\partial\left(h \varepsilon_{n}, h \varepsilon_{n+1}\right), \partial\left(h \varepsilon_{n+1}, h \varepsilon_{n+2}\right), \partial\left(h \wp_{n}, h \wp_{n+1}\right), \partial\left(h \wp_{n+1}, h \wp_{n+2}\right)\right\}\right) \\
& -\hat{\eta}\left(\max \left\{\partial\left(h \varepsilon_{n}, h \varepsilon_{n+1}\right), \partial\left(h \varepsilon_{n+1}, h \varepsilon_{n+2}\right), \partial\left(h \wp_{n}, h \wp_{n+1}\right), \partial\left(h \wp_{n+1}, h \wp_{n+2}\right)\right\}\right),
\end{aligned}
$$


where where

$$
\begin{aligned}
\mathscr{P}(\varepsilon, \wp, \zeta, \Im)= & \max \left\{\frac{\partial(\zeta, \mathscr{I} \partial(\zeta, \Im))[1+\partial(\varepsilon, \mathscr{I} \partial(\varepsilon, \wp))]}{1+\partial(\varepsilon, \zeta)}, \frac{\partial(\varepsilon, \mathscr{I} \partial(\zeta, \Im))+\partial(\zeta, \mathscr{I} \partial(\varepsilon, \wp))}{2 s},\right. \\
& \partial(\varepsilon, \mathscr{I} \partial(\varepsilon, \wp)), \partial(\zeta, \mathscr{I} \partial(\zeta, \Im)), \partial(\varepsilon, \zeta)\} .
\end{aligned}
$$

$$
\Gamma_{n}=\max \left\{\partial\left(h \varepsilon_{n+1}, h \varepsilon_{n+2}\right), \partial\left(h \wp_{n+1}, h \wp_{n+2}\right)\right\} .
$$

Let us denote,

$$
\begin{gathered}
\varkappa_{n}=\max \left\{\partial\left(h \varepsilon_{n}, h \varepsilon_{n+1}\right), \partial\left(h \varepsilon_{n+1}, h \varepsilon_{n+2}\right),\right. \\
\left.\partial\left(h \wp_{n}, h \wp_{n+1}\right), \partial\left(h \wp_{n+1}, h \wp_{n+2}\right)\right\} .
\end{gathered}
$$

Hence from equations (29)-(32), we obtain

$$
s^{k} \Gamma_{n} \leq \varkappa_{n} .
$$

Now to claim that

$$
\Gamma_{n} \leq \lambda \Gamma_{n-1},
$$

for $n \geq 1$ and $\lambda=\frac{1}{s^{k}} \in[0,1)$.

Suppose that if $\varkappa_{n}=\Gamma_{n}$ then from (34), we get $s^{k} \Gamma_{n} \leq \Gamma_{n}$ this leads to $\Gamma_{n}=0$, since $s>1$ and thus (35) holds. Suppose $\varkappa_{n}=\max \left\{\partial\left(h \varepsilon_{n}, h \varepsilon_{n+1}\right), \partial\left(h \wp_{n}, h \wp_{n+1}\right)\right\}$, i.e., $\varkappa_{n}=\Gamma_{n-1}$ then (34) follows (35).

Now from (34), we obtain that $\Gamma_{n} \leq \lambda^{n} \delta_{0}$ and hence,

$$
\partial\left(h \varepsilon_{n+1}, h \varepsilon_{n+2}\right) \leq \lambda^{n} \Gamma_{0} \text { and } \partial\left(h \wp_{n+1}, h \wp_{n+2}\right) \leq \lambda^{n} \Gamma_{0},
$$

which shows that $\left\{h \varepsilon_{n}\right\}$ and $\left\{h \wp_{n}\right\}$ in $\mathscr{E}$ are Cauchy sequences by Lemma 3.1 of [20]. Therefore, we can conclude from Theorem 2.2 of [5] that, $\mathscr{I}$ and $h$ have a coincidence point in $\mathscr{E}$.

Corollary 3.10 Suppose that $(\mathscr{E}, ð, \preceq)$ is a complete partially ordered b-metric space. A continuous mapping $\mathscr{I}: \mathscr{E} \times \mathscr{E} \rightarrow \mathscr{E}$ has a mixed monotone property and is satisfying the below contraction conditions for all $\varepsilon, \wp, \zeta, \Im \in \mathscr{E}$ such that $\varepsilon \preceq \zeta$ and $\wp \succeq \mathfrak{I}, k>2, s>1$, $\check{\psi} \in \hat{\Phi}$ and $\hat{\eta} \in \hat{\Psi}$ :

(i). $\quad \quad \check{\psi}\left(s^{k} \partial(\mathscr{I}(\varepsilon, \wp), \mathscr{I}(\zeta, \mathfrak{I}))\right) \leq \breve{\psi}\left(\mathscr{P}_{h}(\varepsilon, \wp, \zeta, \mathfrak{I})\right)$

$$
-\hat{\eta}\left(\mathscr{P}_{h}(\varepsilon, \wp, \zeta, \mathfrak{I})\right) \text {, }
$$

(ii). $\quad \quad \quad \quad(\mathscr{I}(\varepsilon, \wp), \mathscr{I}(\zeta, \mathfrak{I})) \leq \frac{1}{s^{k}} \mathscr{P}_{h}(\varepsilon, \wp, \zeta, \mathfrak{I})$

$$
-\frac{1}{s^{k}} \hat{\eta}\left(\mathscr{P}_{h}(\varepsilon, \wp, \zeta, \mathfrak{I})\right)
$$

If there exists $\left(\varepsilon_{0}, \wp_{0}\right) \in \mathscr{E} \times \mathscr{E}$ such that $\varepsilon_{0} \preceq \mathscr{I}\left(\varepsilon_{0}, \wp_{0}\right)$ and $\wp_{0} \succeq \mathscr{I}\left(\wp_{0}, \varepsilon_{0}\right)$, then $\mathscr{I}$ has a coupled fixed point in $\mathscr{E}$.

Theorem 3.11 The unique coupled common fixed point for $\mathscr{I}$ and $h$ exists in Theorem 3.9, if for every $(\varepsilon, \wp),(k, \ell) \in \mathscr{E} \times \mathscr{E}$ there exists some $(\Lambda, \Upsilon) \in \mathscr{E} \times \mathscr{E}$ such that $(\mathscr{I}(\Lambda, \Upsilon), \mathscr{I}(\Upsilon, \Lambda))$ is comparable to $(\mathscr{I}(\varepsilon, \wp), \mathscr{I}(\wp, \varepsilon))$ and to $(\mathscr{I}(k, \mathscr{I}), \mathscr{I}(\ell, k))$.

Proof The existence of a coupled coincidence point for $\mathscr{I}$ and $h$ is guaranteed by the Theorem 3.9. Let $(\varepsilon, \wp),(k, \ell) \in \mathscr{E} \times \mathscr{E}$ are two coupled coincidence points of $\mathscr{I}$ and $h$. Now, we assert that $h \varepsilon=h k$ and $h \wp=h \ell$. By the hypotheses $(\mathscr{I}(\Lambda, \Upsilon), \mathscr{I}(\Upsilon, \Lambda))$ is comparable to $(\mathscr{I}(\varepsilon, \wp), \mathscr{I}(\wp, \varepsilon))$ and to $(\mathscr{I}(k, \mathscr{I}), \mathscr{I}(\ell, k))$ for some $(\Lambda, \Upsilon) \in \mathscr{E} \times \mathscr{E}$.

Now, assume the following

$$
\begin{aligned}
& (\mathscr{I}(\varepsilon, \wp), \mathscr{I}(\wp, \varepsilon)) \leq(\mathscr{I}(\Lambda, \Upsilon), \mathscr{I}(\Upsilon, \Lambda)) \\
& \quad \text { and }(\mathscr{I}(k, \ell), \mathscr{I}(\ell, k)) \leq(\mathscr{I}(\Lambda, \Upsilon), \mathscr{I}(\Upsilon, \Lambda)) .
\end{aligned}
$$

Suppose $\Lambda_{0}=\Lambda$ and $\Upsilon_{0}=\Upsilon$ then there is a point $\left(\Lambda_{1}, \Upsilon_{1}\right) \in \mathscr{E} \times \mathscr{E}$ such that

$$
h \Lambda_{1}=\mathscr{I}\left(\Lambda_{0}, \Upsilon_{0}\right), \quad h \Upsilon_{1}=\mathscr{I}\left(\Upsilon_{0}, \Lambda_{0}\right)(n \geq 1)
$$

As by applying the preceding argument repeatedly, we have the sequences $\left\{h \Lambda_{n}\right\}$ and $\left\{h \Upsilon_{n}\right\}$ in $\mathscr{E}$ such that

$$
h \Lambda_{n+1}=\mathscr{I}\left(\Lambda_{n}, \Upsilon_{n}\right), \quad h \Upsilon_{n+1}=\mathscr{I}\left(\Upsilon_{n}, \Lambda_{n}\right)(n \geq 0) .
$$

Define the sequences in the same way $\left\{h \varepsilon_{n}\right\},\left\{\wp_{n}\right\}$ and, $\left\{h k_{n}\right\},\left\{h \ell_{n}\right\}$ in $\mathscr{E}$ by setting $\varepsilon_{0}=\varepsilon, \wp_{0}=\wp$ and $k_{0}=k$, $\ell 0=\ell$. Further, we have that

$$
\begin{aligned}
& \quad h \varepsilon_{n} \rightarrow \mathscr{I}(\varepsilon, \wp), h \wp_{n} \rightarrow \mathscr{I}(\wp, \varepsilon), \\
& \quad h k_{n} \rightarrow \mathscr{I}(k, \ell), h_{\ell} \rightarrow \mathscr{I}(\ell, k)(n \geq 1) .
\end{aligned}
$$

Thus by induction, we get

$$
\left(h \varepsilon_{n}, h \wp_{n}\right) \leq\left(h \Lambda_{n}, h \Upsilon_{n}\right) \text { for every } n \text {. }
$$




$$
\begin{aligned}
\check{\psi}\left(\partial\left(h \varepsilon, h \Lambda_{n+1}\right)\right) \leq \check{\psi}\left(s^{k} \partial\left(h \varepsilon, h \Lambda_{n+1}\right)\right) & =\check{\psi}\left(s^{k} \partial\left(\mathscr{I}(\varepsilon, \wp), \mathscr{I}\left(\Lambda_{n}, \Upsilon_{n}\right)\right)\right) \\
& \leq \breve{\psi}\left(\mathscr{P}_{h}\left(\varepsilon, \wp, \Lambda_{n}, \Upsilon_{n}\right)\right)-\hat{\eta}\left(\mathscr{P}_{h}\left(\varepsilon, \wp, \Lambda_{n}, \Upsilon_{n}\right)\right),
\end{aligned}
$$

where

$$
\begin{aligned}
\mathscr{P}_{h}\left(\varepsilon, \wp, \Lambda_{n}, \Upsilon_{n}\right)= & \max \left\{\frac{\partial\left(h \Lambda_{n}, \mathscr{I} \partial\left(\Lambda_{n}, \Upsilon_{n}\right)\right)[1+\partial(h \varepsilon, \mathscr{I} \partial(\varepsilon, \wp))]}{1+\partial\left(h \varepsilon, h \Lambda_{n}\right)}, \frac{\partial\left(h \varepsilon, \mathscr{I} \partial\left(\Lambda_{n}, \Upsilon_{n}\right)\right)+\partial\left(h \Lambda_{n}, \mathscr{I} \partial(\varepsilon, \wp)\right.}{2 s},\right. \\
& \left.\partial(h \varepsilon, \mathscr{I} \partial(\varepsilon, \wp)), \partial\left(h \Lambda_{n}, \mathscr{I} \partial\left(\Lambda_{n}, \Upsilon_{n}\right)\right), \partial\left(h \varepsilon, h \Lambda_{n}\right)\right\} \\
= & \max \left\{0, \frac{\partial\left(h \varepsilon, h \Lambda_{n}\right)}{s}, \partial\left(h \varepsilon, h \Lambda_{n}\right)\right\}=\partial\left(h \varepsilon, h \Lambda_{n}\right) .
\end{aligned}
$$

Therefore from (39), we have

$$
\lim _{n \rightarrow+\infty} \partial\left(h \varepsilon, h \Lambda_{n}\right)=0 \text { and } \lim _{n \rightarrow+\infty} \partial\left(h \wp, h \Upsilon_{n}\right)=0 .
$$

$$
\check{\psi}\left(ð\left(h \varepsilon, h \Lambda_{n+1}\right)\right) \leq \check{\psi}\left(\partial\left(h \varepsilon, h \Lambda_{n}\right)\right)-\hat{\eta}\left(\partial\left(h \varepsilon, h \Lambda_{n}\right)\right) .
$$

As by the similar argument, we acquire that

From the similar argument as above, we obtain that

$$
\check{\psi}\left(\partial\left(h \wp, h \Upsilon_{n+1}\right)\right) \leq \check{\psi}\left(ð\left(h \wp, h \Upsilon_{n}\right)\right)-\hat{\eta}\left(ð\left(h \wp, h \Upsilon_{n}\right)\right)
$$

$$
\lim _{n \rightarrow+\infty} \partial\left(h k, h \Lambda_{n}\right)=0 \text { and } \lim _{n \rightarrow+\infty} \partial\left(h \mathscr{I}, h \Upsilon_{n}\right)=0 .
$$

Hence from (40) and (41), we have

Therefore from (44) and (45), we get $h \varepsilon=h k$ and $h \wp=h \mathscr{I}$. Since $h \varepsilon=\mathscr{I}(\varepsilon, \wp)$ and $h \wp=\mathscr{I}(\wp, \varepsilon)$ and, the commutative property of $\mathscr{I}$ and $h$ implies that

$$
\begin{aligned}
\check{\psi}\left(\max \left\{\partial\left(h \varepsilon, h \Lambda_{n+1}\right), \partial\left(h \wp, h \Upsilon_{n+1}\right)\right\}\right) \leq & \breve{\psi}\left(\max \left\{\partial\left(h \varepsilon, h \Lambda_{n}\right), \partial\left(h \wp, h \Upsilon_{n}\right)\right\}\right) \\
& -\hat{\eta}\left(\max \left\{\partial\left(h \varepsilon, h \Lambda_{n}\right), \partial\left(h \wp, h \Upsilon_{n}\right)\right\}\right) \\
& <\breve{\psi}\left(\max \left\{\partial\left(h \varepsilon, h \Lambda_{n}\right), \partial\left(h \wp, h \Upsilon_{n}\right)\right\}\right) .
\end{aligned}
$$

Thus the property of $\breve{\psi}$ implies,

$$
\begin{array}{r}
\max \left\{\partial\left(h \varepsilon, h \Lambda_{n+1}\right), \partial\left(h \wp, h \Upsilon_{n+1}\right)\right\} \\
<\max \left\{\partial\left(h \varepsilon, h \Lambda_{n}\right), \partial\left(h \wp, h \Upsilon_{n}\right)\right\} .
\end{array}
$$

Hence, $\max \left\{\partial\left(h \varepsilon, h \Lambda_{n}\right), \partial\left(h \wp, h \Upsilon_{n}\right)\right\}$ is a decreasing sequence of positive reals and bounded below and by a result, we have

$$
\lim _{n \rightarrow+\infty} \max \left\{\partial\left(h \varepsilon, h \Lambda_{n}\right), \partial\left(h \wp, h \Upsilon_{n}\right)\right\}=\Gamma, \Gamma \geq 0 .
$$

Therefore as $n \rightarrow+\infty$ in equation (42), we get

$$
\breve{\psi}(\Gamma) \leq \breve{\psi}(\Gamma)-\hat{\eta}(\Gamma),
$$

from which we get $\hat{\eta}(\Gamma)=0$, this implies that $\Gamma=0$. Therefore,

$$
\lim _{n \rightarrow+\infty} \max \left\{\partial\left(h \varepsilon, h \Lambda_{n}\right), \partial\left(h \wp, h \Upsilon_{n}\right)\right\}=0 .
$$

$$
\begin{aligned}
& h(h \varepsilon)=h(\mathscr{I}(\varepsilon, \wp))=\mathscr{I}(h \varepsilon, h \wp) \text { and } h(h \wp) \\
& \quad=h(\mathscr{I}(\wp, \varepsilon))=\mathscr{I}(h \wp, h \varepsilon) .
\end{aligned}
$$

If $h \varepsilon=\Lambda^{*}$ and $h \wp=\Upsilon^{*}$, then from (46), we get

$$
h(\Lambda)=\mathscr{I}\left(\Lambda^{*}, \Upsilon^{*}\right) \text { and } h\left(\Upsilon^{*}\right)=\mathscr{I}\left(\Upsilon^{*}, \Lambda^{*}\right),
$$

which exhibits that $\left(\Lambda^{*}, \Upsilon^{*}\right)$ is a coupled coincidence point of $\mathscr{I}, h$. Hence, $h\left(\Lambda^{*}\right)=h k$ and $h\left(\Upsilon^{*}\right)=h \mathscr{I}$ which in turn gives that $h(\Lambda)=\Lambda^{*}$ and $h\left(\Upsilon^{*}\right)=\Upsilon^{*}$. Therefore from (47), $\left(\Lambda^{*}, \Upsilon^{*}\right)$ is a coupled common fixed point of $\mathscr{I}, h$.

Let $\left(\Lambda_{1}^{*}, \Upsilon_{1}^{*}\right)$ be another coupled common fixed point of $\mathscr{I}, h$. Then, $\Lambda_{1}^{*}=h \Lambda_{1}^{*}=\mathscr{I}\left(\Lambda_{1}^{*}, \Upsilon_{1}^{*}\right)$ and $\Upsilon_{1}^{*}=h \Upsilon_{1}^{*}=\mathscr{I}\left(\Upsilon_{1}^{*}, \Lambda_{1}^{*}\right)$. But $\left(\Lambda_{1}^{*}, \Upsilon_{1}^{*}\right)$ is a coupled common fixed point of $\mathscr{I}$ and $h$ then, $h \Lambda_{1}^{*}=h \varepsilon=\Lambda$ and $h \Upsilon_{1}^{*}=h \wp=\Upsilon^{*}$. Therefore, $\Lambda_{1}^{*}=h \Lambda_{1}^{*}=h \Lambda=\Lambda$ and $\Upsilon_{1}^{*}=h \Upsilon_{1}^{*}=h \Upsilon^{*}=\Upsilon^{*}$. Hence the uniqueness. 
Theorem 3.12 In Theorem 3.11, if $h \varepsilon_{0} \preceq h \wp_{0}$ or $h \varepsilon_{0} \succeq h \wp_{0}$, then a unique common fixed point of $\mathscr{I}$ and $h$ can be found.

Proof Assume that $(\varepsilon, \wp) \in \mathscr{E}$ is a unique coupled common fixed point of $\mathscr{I}$ and $h$. Then to demonstrate that $\varepsilon=\wp$. Suppose that $h \varepsilon_{0} \preceq h \wp_{0}$, then we get by induction that, $h \varepsilon_{n} \preceq h \wp_{n}$ for $n \geq 0$. From Lemma 2 of [21], we have
A self-mapping $\mathscr{I}$ on $\mathscr{E}$ defined by $\mathscr{I} e_{1}=\mathscr{I} e_{2}=\mathscr{I} e_{3}=\mathscr{I} e_{4}=\mathscr{I} e_{5}=1, \mathscr{I} e_{6}=2$ has a fixed point with $\breve{\psi}(y)=\frac{y}{2}$ and $\hat{\eta}(y)=\frac{y}{4}$ where $y \in[0,+\infty)$.

Proof When $s=2,(\mathscr{E}, \widetilde{\partial}, \leq)$ is a complete partially ordered $b$-metric space. Let $\varepsilon, \wp \in \mathscr{E}$ such that $\varepsilon<\wp$ then we'll look at the cases below.

$$
\begin{aligned}
& \check{\psi}\left(s^{k-2} \partial(\varepsilon, \wp)\right)=\check{\psi}\left(s^{k} \frac{1}{s^{2}} \partial(\varepsilon, \wp)\right) \leq \lim _{n \rightarrow+\infty} \sup \check{\psi}\left(s^{k} ð\left(\varepsilon_{n+1}, \wp_{n+1}\right)\right) \\
& =\lim _{n \rightarrow+\infty} \sup \check{\psi}\left(s^{k} \partial\left(\mathscr{I}\left(\varepsilon_{n}, \wp_{n}\right), \mathscr{I}\left(\wp_{n}, \varepsilon_{n}\right)\right)\right) \\
& \leq \lim _{n \rightarrow+\infty} \sup \check{\psi}\left(\mathscr{P}_{h}\left(\varepsilon_{n}, \wp_{n}, \wp_{n}, \varepsilon_{n}\right)\right)-\lim _{n \rightarrow+\infty} \inf \hat{\eta}\left(\mathscr{P}_{h}\left(\varepsilon_{n}, \wp_{n}, \wp_{n}, \varepsilon_{n}\right)\right) \\
& \leq \check{\psi}(ð(\varepsilon, \wp))-\lim _{n \rightarrow+\infty} \inf \hat{\eta}\left(\mathscr{P}_{h}\left(\varepsilon_{n}, \wp_{n}, \wp_{n}, \varepsilon_{n}\right)\right) \\
& <\breve{\psi}(ð(\varepsilon, \wp)) \text {, }
\end{aligned}
$$

a contradiction. Hence, $\varepsilon=\wp$.

The result can also be similar in the case of $h \varepsilon_{0} \succeq h \wp_{0}$.

Remark 3.13 While $s=1$ and the result of [19], the condition

$$
\begin{aligned}
& \check{\psi}(\partial(\mathscr{I}(\varepsilon, \wp), \mathscr{I}(\partial, \mathfrak{I}))) \leq \check{\psi}(\max \{\partial(h \varepsilon, h \partial), \partial(h \wp, h \mathfrak{I})\}) \\
& \quad-\hat{\eta}(\max \{\partial(h \varepsilon, h \partial), \partial(h \wp, h \mathfrak{I})\})
\end{aligned}
$$

is equivalent to,

$$
\partial(\mathscr{I}(\varepsilon, \wp), \mathscr{I}(ð, \mathfrak{I})) \leq \varphi(\max \{\partial(h \varepsilon, h \partial), \partial(h \wp, h \mathfrak{I})\}),
$$

where $\check{\psi} \in \hat{\Phi}, \hat{\eta} \in \hat{\Psi}$ and $\varphi$ is a continuous self mapping on $[0,+\infty)$ with $\varphi(y)<y$ for every $y>0$ with $\varphi(y)=0$ if and only if $y=0$. Hence the results found here are generalized and extended the results of $[11,18,22,25,27]$ and several comparable results.

Now depending on the type of a metric, some examples are shown here under.

Example 3.14 Let $\mathscr{E}=\left\{e_{1}, e_{2}, e_{3}, e_{4}, e_{5}, e_{6}\right\} \quad$ and ठ : $\mathscr{E} \times \mathscr{E} \rightarrow \mathscr{E}$ be a metric defined by
Case 1. If $\varepsilon, \wp \in\left\{e_{1}, e_{2}, e_{3}, e_{4}, e_{5}\right\} \quad$ then $\partial(\mathscr{I} \varepsilon, \mathscr{I} \wp)=\partial\left(e_{1}, e_{1}\right)=0$. Hence,

$$
\check{\psi}(2 ð(\mathscr{I} \varepsilon, \mathscr{I} \wp))=0 \leq \check{\psi}(\mathscr{P}(\varepsilon, \wp))-\hat{\eta}(\mathscr{P}(\varepsilon, \wp)) .
$$

Case 2. If $\varepsilon \in\left\{e_{1}, e_{2}, e_{3}, e_{4}, e_{5}\right\}$ and $\wp=e_{6}$, then $\partial(\mathscr{I} \varepsilon, \mathscr{I} \wp)=\partial\left(e_{1}, e_{2}\right)=3, \quad \mathscr{P}\left(e_{6}, e_{5}\right)=20 \quad$ and $\mathscr{P}\left(\varepsilon, e_{6}\right)=12$, for $\varepsilon \in\left\{e_{1}, e_{2}, e_{3}, e_{4}\right\}$. Hence,

$$
\check{\psi}(2 ð(\mathscr{I} \varepsilon, \mathscr{I} \wp)) \leq \frac{\mathscr{P}(\varepsilon, \wp)}{4}=\check{\psi}(\mathscr{P}(\varepsilon, \wp))-\hat{\eta}(\mathscr{P}(\varepsilon, \wp)) .
$$

As a result, all of the conditions of Theorem 3.1 are met, and hence $\mathscr{I}$ has a fixed point.

Example 3.15 Let us define a metric $ð$ with usual order $\leq$ by

$$
\partial(\varepsilon, \wp)= \begin{cases}0, & \text { if } \varepsilon=\wp \\ 1, & \text { if } \varepsilon \neq \wp \in\{0,1\} \\ |\varepsilon-\wp|, & \text { if } \varepsilon, \wp \in\left\{0, \frac{1}{2 n}, \frac{1}{2 m}: n \neq m \geq 1\right\} \\ 6, & \text { otherwise. }\end{cases}
$$

where $\mathscr{E}=\left\{0,1, \frac{1}{2}, \frac{1}{3}, \frac{1}{4}, \ldots, \frac{1}{n}, \ldots\right\}$. A self-mapping $\mathscr{I}$ on $\mathscr{E}$ by $\mathscr{I} 0=0, \mathscr{I} \frac{1}{n}=\frac{1}{12 n}(n \geq 1)$ has a fixed point with $\check{\psi}(y)=y$ and $\hat{\eta}(y)=\frac{4 y}{5}$ for $y \in[0,+\infty)$.

$$
\begin{aligned}
& (\varepsilon, \wp)=(\wp, \varepsilon)=0, \text { if } \varepsilon=\wp=\left\{e_{1}, e_{2}, e_{3}, e_{4}, e_{5}, e_{6}\right\} \\
& \text { and } \varepsilon=\wp,(\varepsilon, \wp)=(\wp, \varepsilon)=3 \text {, if } \varepsilon=\wp=\left\{e_{1}, e_{2}, e_{3}, e_{4}, e_{5}\right\} \\
& \text { and } \varepsilon \neq \wp,(\varepsilon, \wp)=(\wp, \varepsilon)=12, \text { if } \varepsilon=\left\{e_{1}, e_{2}, e_{3}, e_{4}\right\} \\
& \text { and } \wp=e_{6},(\varepsilon, \wp)=(\wp, \varepsilon)=20, \text { if } \varepsilon=e_{5} \text { and } \wp=e_{6}, \text { with usual order } \leq .
\end{aligned}
$$


Proof $\partial$ is clearly discontinuous, and $(\mathscr{E}, \partial, \leq)$ is a complete partially ordered $b$-metric space for $s=\frac{12}{5}$. Now we'll look at the following cases for $\varepsilon, \wp \in \mathscr{E}$ with $\varepsilon<\wp$.

Case 1. Suppose $\varepsilon=0$ and $\wp=\frac{1}{n}(n>0)$, then $\partial\left(\mathscr{I}_{\varepsilon}, \mathscr{I}_{\wp}\right)=\check{\partial}\left(0, \frac{1}{12 n}\right)=\frac{1}{12 n} \quad$ and $\quad \mathscr{P}(\varepsilon, \wp)=\frac{1}{n} \quad$ and $\mathscr{P}(\varepsilon, \wp)=\{1,6\}$. Thus,

$$
\check{\psi}\left(\frac{12}{5} ð(\mathscr{I} \varepsilon, \mathscr{I} \wp)\right) \leq \frac{\mathscr{P}(\varepsilon, \wp)}{5}=\check{\psi}(\mathscr{P}(\varepsilon, \wp))-\hat{\eta}(\mathscr{P}(\varepsilon, \wp)) .
$$

Case 2. Let $\varepsilon=\frac{1}{m}$ and $\wp=\frac{1}{n}$ where $m>n \geq 1$, then

$$
ð(\mathscr{I} \varepsilon, \mathscr{I} \wp)=ð\left(\frac{1}{12 m}, \frac{1}{12 n}\right), \mathscr{P}(\varepsilon, \wp) \geq \frac{1}{n}-\frac{1}{m} \text { or } \mathscr{P}(\varepsilon, \wp)=6 .
$$

Thus,

$$
\check{\psi}\left(\frac{12}{5} \check{\partial}\left(\mathscr{I} \varepsilon, \mathscr{I}_{\wp}\right)\right) \leq \frac{\mathscr{P}(\varepsilon, \wp)}{5}=\check{\psi}(\mathscr{P}(\varepsilon, \wp))-\hat{\eta}(\mathscr{P}(\varepsilon, \wp)) .
$$

Hence, we have the conclusion from Theorem 3.1 as all assumptions are fulfilled.

Example 3.16 Define a metric $d: \mathscr{E} \times \mathscr{E} \rightarrow \mathscr{E}$, where $\mathscr{E}=\left\{\tilde{\ell} / \tilde{\ell}:\left[a_{1}, a_{2}\right] \rightarrow\left[a_{1}, a_{2}\right]\right.$ is continuous $\}$ by

$$
\partial\left(\tilde{\ell}_{1}, \tilde{\ell}_{2}\right)=\sup _{y \in\left[a_{1}, a_{2}\right]}\left\{\left|\tilde{\ell}_{1}(y)-\tilde{\ell}_{2}(y)\right|^{2}\right\}
$$

for any $\tilde{\ell}_{1}, \tilde{\ell}_{2} \in \mathscr{E}, \quad 0 \leq a_{1}<a_{2}$ with $\tilde{\ell}_{1} \preceq \tilde{\ell}_{2}$ implies $a_{1} \leq \tilde{\ell}_{1}(y) \leq \tilde{\ell}_{2}(y) \leq a_{2,} y \in\left[a_{1}, a_{2}\right]$. A self-mapping $\mathscr{I}$ on $\mathscr{E}$ defined by $\mathscr{I} \tilde{\tilde{\ell}}=\frac{\ell}{5}, \tilde{\ell} \in \mathscr{E}$ has a unique fixed point with $\breve{\psi}(y)=y$ and $\hat{\eta}(y)=\frac{y}{3}$ for any $y \in[0,+\infty]$.

Proof $\operatorname{As} \min \left(\tilde{\ell}_{1}, \tilde{\ell}_{2}\right)(y)=\min \left\{\tilde{\ell}_{1}(y), \tilde{\ell}_{2}(y)\right\}$ is continuous and all other assumptions of Theorem 3.3 are fulfilled for $s=2$. Hence, $0 \in \mathscr{E}$ is a unique fixed point of $\mathscr{I}$.

\section{Limitations}

We examined a fixed point, a coincidence point and a couple coincidence point for mappings that are satisfying generalized $(\breve{\psi}, \hat{\eta})$-weak contractions in a partially ordered $b$-metric space. The findings in this paper are generalized and extended a few well-known results in the current literature. Some examples are shown at the end to support the results obtained here.

\section{Acknowledgements}

The authors do thankful to the editor and anonymous reviewers for their valuable suggestions and comments which improved the contents of the paper.

\section{Authors' contributions}

NSR contributed in the conceptualization, formal analysis, methodology, writing, editing and approving the manuscript. KK involved in formal analysis, methodology and writing the original draft. KP supervised the work and critically revised the manuscript. All authors read and approved the final manuscript.

\section{Funding}

Not applicable.

\section{Availability of data and materials}

Not applicable.

\section{Declarations}

Ethics approval and consent to participate

Not applicable.

\section{Consent for publication}

Not applicable.

\section{Competing interests}

The authors declare that they have no competing interests.

\section{Author details}

${ }^{1}$ Department of Applied Mathematics, School of Applied Natural Sciences, Adama Science and Technology University, Post Box No.1888, Adama, Ethiopia. ${ }^{2}$ Department of Mathematics, Vignan's Foundation for Science, Technology \& Research, Vadlamudi 522213, Andhra Pradesh, India. ${ }^{3}$ Department of Applied Mathematics, Koneru Lakshmaiah Educational Foundation, Vaddeswaram, Andhra Pradesh, India.

Received: 25 March 2021 Accepted: 9 June 2021

Published online: 08 July 2021

\section{References}

1. Abas M, Ali B, Nazir T, Dedović NM, Bin-Mohsin B, Radenović SN. Solutions and Ulam-Hyers stability of differential inclusions involving Suzuki type multivalued mappings on b-metric spaces. Vojnotehnički Glasnik. 2020;68(3):438-87.

2. Abdou AA, Cho YJ, Saadati R. Distance type and common fixed point theorems in Menger probabilistic metric type spaces. Appl Math Comput. 2015;265:1145-54. https://doi.org/10.1016/j.amc.2015.05.052.

3. Agarwal RP, El-Gebeily MA, O'Regan D. Generalized contractions in partially ordered metric spaces. Appl Anal. 2008;87:1-8. https://doi.org/ 10.1080/00036810701714164.

4. Aghajani A, Arab R. Fixed points of $(\psi, \phi, \theta)$-contractive mappings in partially ordered $b$-metric spaces and applications to quadratic integral equations. Fixed Point Theory Appl. 2013;2013(1):1-20.

5. Akkouchi M. Common fixed point theorems for two self mappings of a b-metric space under an implicit relation. Hacet J Math Stat. 2011;40(6):805-10.

6. Aleksić S, Huaping H, Mitrović ZD, Radenović S. Remarks on some fixed point results in b-metric spaces. J Fixed Point Theory Appl. 2018;20:147.

7. Allahyari R, Arab R, Haghighi AS. A generalization on weak contractions in partially ordered $b$-metric spaces and its applications to quadratic integral equations. J Inequal Appl. 2014;2014(1):1-5.

8. Aydi H, Dedović N, Bin-Mohsin B, Filipović M, Radenović S. Some new observations on Geraghty and Ćirić type results in b-metric spaces. Mathematics. 2019;7:643. https://doi.org/10.3390/math707064.

9. Bakhtin IA. The contraction principle in quasimetric spaces. Func An Ulianowsk Gos Fed Ins. 1989;30:26-37.

10. Mituku Belay. Kalyani K, Seshagiri Rao N, Some fixed point results of generalized $(\phi, \psi)$-contractive mappings in ordered $b$-metric spaces. BMC Res Notes. 2020;13:537. https://doi.org/10.1186/s13104-020-05354-1. 
11. Bhaskar TG, Lakshmikantham V. Fixed point theorems in partially ordered metric spaces and applications. Nonlinear Anal. 2006;65:1379-93. https:// doi.org/10.1016/j.na.2005.10.017.

12. Chandok S, Karapinar E. Common fixed point of generalized rational type contraction mappings in partially ordered metric spaces. Thai J Math. 2013;11(2):251-60.

13. Kongban Chayut, Kumam Poom. Quadruple random common fixed point results of generalized Lipschitz mappings in cone $b$-metric spaces over Banach algebras. J Nonlinear Sci Appl. 2018;11:131-49. https://doi. org/10.22436/jnsa.011.01.10.

14. Czerwik S. Contraction mappings in b-metric spaces. Acta Math Univ Ostrav. 1993;1:5-11.

15. Czerwik S. Nonlinear set-valued contraction mappings in b-metric spaces. Atti Semin Mat Fis Univ. 1998;46(2):263-76.

16. Demma M, Saadati R, Vetro P. Multi-valued operators with respect wt-distance on metric type spaces. Bull Iranian Math Soc. 2016;42(6):1571-82.

17. Gehad M. Abd-Elhamed, Fixed point results for $(\beta, \alpha)$-implicit contractions in two generalized $b$-metric spaces. J Nonlinear Sci Appl. 2021;14:39-47. https://doi.org/10.22436/jnsa.014.01.05.

18. Faraji Hamid, Savić Dragana, Radenović S. Fixed point theorems for Geraghty contraction type mappings in $b$-metric spaces and applications. Aximos. 2019:8:34

19. Jachymski J. Equivalent conditions for generalized contractions on (ordered) metric spaces. Nonlinear Anal. 2011;74:768-74.

20. Jovanović M, Kadelburg Z, Radenović S. Common fixed point results in metric-type spaces. Fixed Point Theory Appl. 2010. https://doi.org/10. 1155/2010/978121.

21. Karapinar E, Mitrović D, Özturk A, Radenović S. On a theorem of Ćirić in b-metric spaces. Rendiconti del Circolo Matematico di Palermo Series 2. https://doi.org/10.1007/s12215-020-00491-9.

22. Lakshmikantham V, Ćirić $\sqcup$. Coupled fixed point theorems for nonlinear contractions in partially ordered metric spaces. Nonlinear Anal. 2009;70:4341-9. https://doi.org/10.1016/j.na.2008.09.020.

23. Mishra LN, Dewangan V, Mishra VN, Karateke S. Best proximity points of admissible almost generalized weakly contractive mappings with rational expressions on b-metric spaces. J Math Computer Sci. 2021;22:97-109.

24. Ye Lingjuan, Shen Congcong. Weakly $(s, r)$-contractive multi-valued operators on b-metric space. J Nonlinear Sci Appl. 2018;11:358-67. https://doi.org/10.22436/jnsa.011.03.04.

25. Luong NV, Thuan NX. Coupled fixed point theorems in partially ordered metric spaces. Bull Math Anal Appl. 2010;4:16-24.

26. Demma Marta, Saadati Reza, Vetro Pasquale. Fixed Point Results on b-Metric Space via Picard Sequences and b-Simulation Functions. Iran J Math Sci Inform. 2016;11(1):123-36. https://doi.org/10.7508/ijmsi.2016.01.011.

27. Hieu Nguyen T, Dung Nguyen V. Some fixed point results for generalized rational type contraction mappings in partially ordered $b$-metric space. Facta Univ Ser Math Inf. 2015:30(1):49-66.
28. Nieto JJ, Loṕez RR. Contractive mapping theorems in partially ordered sets and applications to ordinary differential equations. Order. 2005;22:223-39. https://doi.org/10.1007/s11083-005-9018-5.

29. Nieto JJ, Loṕez RR. Existence and uniqueness of fixed point in partially ordered sets and applications to ordinary differential equations. Acta Math Sinica Engl Ser. 2007;23(12):2205-12. https://doi.org/10.1007/ s10114-005-0769-0.

30. O'Regan D, Petrutel A. Fixed point theorems for generalized contractions in ordered metric spaces. J Math Anal Appl. 2008;341:1241-52.

31. Lohawech Panisa, Kaewcharoen Anchalee. Fixed point theorems for generalized JS-quasi-contractions in complete partial $b$-metric spaces. J Nonlinear Sci Appl. 2019;12:728-39. https://doi.org/10.22436/jnsa.012.11.04.

32. Ran ACM, Reurings MCB. A fixed point theorem in partially ordered sets and some applications to matrix equations. Proc Am Math Soc. 2004;132:1435-43. https://doi.org/10.1090/S0002-9939-03-07220-4.

33. Seshagiri Rao N, Kalyani K. Fixed point theorems for nonlinear contractive mappings in ordered $b$-metric space with auxiliary function. BMC Res Notes. 2020;13:451. https://doi.org/10.1186/s13104-020-05273-1.

34. Seshagiri Rao N, Kalyani K. Generalized contractions to coupled fixed point theorems in partially ordered metric spaces. SibFU. 2020;13(4):492502. 10.17516/1997-1397-2020-13-4-492-502.

35. Seshagiri Rao N, Kalyani K. Coupled fixed point theorems with rational expressions in partially ordered metric spaces. J Anal. 2020;28(4):1085-95. https://doi.org/10.1007/s41478-020-00236-y.

36. Seshagiri Rao N, Kalyani K, Kejal K. Contractive mapping theorems in partially ordered metric spaces. CUBO. 2020;22(2):203-14.

37. Seshagiri Rao N, Kalyani K. Unique fixed point theorems in partially ordered metric spaces. Heliyon. 2020;6(11):e05563. https://doi.org/10. 1016/j.heliyon.2020.e05563.

38. Suzuki T. Basic inequality on a b-metric space and its applications. J Inequal Appl. 2017. https://doi.org/10.1186/s13660-017-1528-3.

39. Suzuki T. Fixed point theorems for single-and set-valued $F$-contractions in b-metric spaces. J Fixed Point Theory Appl. 2018;20(1):12. https://doi.org/ 10.1007/s11784-018-0519-4.

40. Antal Swati, Gairola uc. Generalized Suzuki type $\alpha-Z$-contraction in $b$ -metric space. J Nonlinear Sci Appl 2020;13:212-22. https://doi.org/10. 22436/jnsa.013.04.06.

41. Xianbing $\mathrm{Wu}$, Zhao Leina. Fixed point theorems for generalized $\alpha-\psi$ type contractive mappings in $b$-metric spaces and applications. J Math Computer Sci. 2018;18:49-62. https://doi.org/10.22436/jmcs.018.01.06.

\section{Publisher's Note}

Springer Nature remains neutral with regard to jurisdictional claims in published maps and institutional affiliations.
Ready to submit your research? Choose BMC and benefit from:

- fast, convenient online submission

- thorough peer review by experienced researchers in your field

- rapid publication on acceptance

- support for research data, including large and complex data types

- gold Open Access which fosters wider collaboration and increased citations

- maximum visibility for your research: over $100 \mathrm{M}$ website views per year

At $\mathrm{BMC}$, research is always in progress.

Learn more biomedcentral.com/submissions 